\title{
Reduction of 3D Spectral Data, Obtained with the 6-meter Telescope
}

\author{
V. V. Vlasiouk
}

Special Astrophysical Observatory of Russian Academy of Sciences, Nyzhnij Arkhyz, Russia,357147

\section{Introduction}

Development of 3D spectral methods on the 6-meter telescope prompted the necessity of creating specialized software for data reduction, adapted to personal computers IBM AT $/ 386 / 486$ class. We had to concentrate our efforts on preparing the software for bidimensional spectral data produced by Multi-Pupil Integral Field Spectrograph of the 6-meter telescope (MPFS) and analog of panoramic spectrophotometer with spectral channeling, using scanning FabryPerot interferometer (PYTHEAS-6).

\section{MPFS data reducing}

MPFS - analog of spectrograph TIGER (Courtes et al., 1988), developed for the 6-meter telescope, provides bidimensional spectroscopy of extended sources, allowing the simultaneous detection of 99 individual spectra, produced by a lens raster from 9 by 11 microlenses. The main features of this instrument are described in (Afanasiev et al, 1994).

The reduction of MPFS data involves the following steps :

- data flat-fielding (applies, if detector's nonuniformities exceeds 2-3 \%);

- identification of individual spectra on the frame and its extraction by integrating along curved trajectories (by direct accumulation of flux in an aperture, or by decomposition of cross-dispersion profiles);

- transformation of extracted spectra to a uniform wavelength scale;

- correction of individual spectra for differences in microlens transmission and flux vignetting through microlens array;

- measurements of astrophysical parameters through all spectra (velocity and intensity maps, etc.).

\subsection{Flat-flelding of spectral data}

It is well-known that all kinds of photon detectors based on image intensifier technology suffer from instabilities depending on time, orientation of system in Earth's magnetic field and other factors. Such instabilities providing shifts of frames up to some pixels render useless the ordinary procedure of flat-fielding. 
In order to suppress nonuniformities present in spectral data as well as in flatfield frames, we decided to use bidimensional cross-correlations, built inside each pair of corresponding fragments from $64 \times 64$ data pixels. The position of the maximum of cross-correlation, yields, according to its definition, the value of a vector, characterizing mutual shift of the accumulation inside both analyzed fragment. The total field of such vectors, being smoothed, describes relative shift of both accumulations and allows to match it for following correction.

\section{PYTHEAS-6 data reduction}

PYTHEAS-6 is a microlens integral field spectrometer, including a scanning Fabry-Perot interferometer, which is illuminated by a sharp angular beam, being mounted on the optical axis between an enlarger and a multi-lens array.

Combined with an ordinary nebular spectrograph with grating or prism as disperser, it allows to detect separate spectra from different parts of an extended sources with a spectral resolution, defined only by the interferential etalon, in a relatively wide spectral range, limited by the detector size. The concept of this instrument has been proposed by le Coarer et al. 1992.

Observations with PYTHEAS consist in a sequence of images for each spectral channel of the etalon, obtained with different distances between its plates. Being modulated by the etalon transparency function, the individual spectra appear as sets of spots with various brightnesses, corresponding to the spectral intensity in the current transparency range. When scanning the spectra by changing the distance between the etalon plates, the location of the spots also changes - they are shifting along dispersion direction according to the main law of the Fabry-Perot.

\subsection{Main Steps of Data Reduction}

Below we describe the principal steps of data reduction on an example of specified individual spectrum.

Integrating a specified spectrum. We integrate along curved trajectories, (quite alike the procedure described in Sec. 2), the specified spectrum through spectral channel images, corresponding to all scanning steps in a complete cycle and aligning the obtained vector data one under another. The resulting image contains the complete spectral information, since upon scanning the full free spectral range of the etalon has been covered. Due to the shift mentioned above, spectral orders are building here inclined tracks. Integrating the signal along these tracks will allow to get the complete spectral information after wavelength calibration. Such a procedure is applied to the integration of the observed object, and continuum and line spectral sources, that provide determination of interference order numbers and wavelength scale.

Data Calibration. Let us define as Wave $(I)$ the wavelength of the $I$-th line of the comparison spectrum. Comparing spectral lines positions with location of tracks for the corresponding continuous spectrum allows to assign them "observed" interference orders, differing from "true" ones by some offset value. Moreover, a number of scanning steps, those where the line intensity has a 
maximum value, give the rational part of this "observed" order Order $(I)$. The calibration procedure consists of :

- forming of so-called the so-called "empirical phase curve" : dependence of products $\operatorname{Order}(I) * W a v e(I)$ as a function of wavelength. This function is approximated by a second or third order polynom.

- finding the relation, allowing to compute for each specified wavelength the corresponding value of interference order and spectral channel.

- sampling the analyzed spectrum with the appropriate step.

Knowing the main law of the Fabry-Perot etalon, it is very easy to show about the "empirical phase curve", that :

- the coefficient of zeroth order in its approximation is, in fact, the so-called "etalon constant", or right side of etalon's equation.

- the coefficient of first order is the "offset" value (i.e., difference between "observed" and "true" values of interference order).

- the coefficient of orders higher than one, describe the phase shift, i.e. the variation of "etalon constant" ( in fact, characteristics of plates) with wavelength.

This procedure reveals the possibility not only to reconstruct PYTHEAS spectra with the resolution defined by the parameters of the FP etalon, but also to determine these parameters from spectral data themselves.

\section{Conclusion}

The application of algorithms which were briefly overviewed to real astrophysical data obtained during observations with PYTHEAS-6 on the 6-meter telescope shows the great potential possibilities of this method for solving problems for which high spectral (better, than $1 \AA$ ) and angular resolution (about $1^{\prime \prime}$ ) are both necessary.

Acknowledgments. The author thanks V.L. Afanasiev, S.N. Dodonov, J. Boulesteix and E. le Coarer for their help and fruitful discussions.

\section{References}

Afanasiev, V.L., Dodonov, S.N., Drabek, S.V., Vlasjuk, V.V., this Colloquim, 1994

le Coarer, E., Georgelin, Y., Monnet, G., 1992, C.R.Acad.Sci.Paris, 315,45.

Courtes, G., Georgelin, Y., Bacon, R., Monnet, G., Boulesteix, J. 1988, in Instrumentation for Ground-Based Optical Astronomy : Present and Future, ed. L. Robinson, 266. 